\section{EPOS2020 and beyond}

The April issue of Rhinology includes the Executive Summary of EPOS2020, highlighting the most important up-dates on disease definitions, treatment algorithms and integrated care pathway approaches of CRS. EPOS2020 (1) is the result of major efforts by a team of passionate rhinologists and associate health care providers coming from all corners of the world coordinated by Professors Wytske Fokkens and Valerie Lund. The Executive Summary presented here illustrates the most important graphs for use in daily practice and teaching purposes.

In addition, the April issue of Rhinology also brings relevant CRS-related aspects to the attention of the readers of Rhinology. The socio-economic impact of CRS has not been extensively studied, and has been evaluated in the UK by the team of Carl Philpott. Wahid et al. ${ }^{(2)}$ here report on the direct and indirect costs of CRS, with an average of 18,7 missed workdays per year in their patient population. As expected, the indirect costs largely exceed the direct costs, and position CRS amongst relevant conditions for health authorities.

As far as immune pathology of CRSwNP is concerned, systemic and local IgE against micro-organisms have been reported in CRSwNP earlier ${ }^{(3)}$. Here, Ohki et al. ${ }^{(4)}$ present their study on fungus-specific lgE in serum of $23 \%$ of patients with CRSwNP, and in nasal polyp tissue of the majority of CRSwNP patients. The presence of fungus-specific lgE points towards an involvement of fungal exposure in the inflammatory pathology of CRSwNP, without proven causality.

Smell impairment gains more attention as major and impactful burden of CRS, and several studies have evaluated the impact of smell capacity in relation to ageing and other factors. Firstly, Stöbauer et al. ${ }^{(5)}$ compared 5 large, population-based studies, and report differences in the prevalence of normosmia within each age and gender specific stratum, despite the use of the same system to test smell function across studies. Four factors considerably contribute to the explanation of prevalence differences, i.e. age, gender, educational level and comorbidity burden. On a separate note, the yearly periodicity of patient presentations at a specialized smell and taste Clinic in Dresden is an interesting observation ${ }^{(6)}$. The seasonal peak in patient presentation in Spring only allows hypothesizing on the reason for this observation, hence warranting further research. Zhang and coworkers ${ }^{(7)}$ demonstrate better smell capacities in physically active elderly CRS patients than in non-exercising elderly, with specific types of exercise being important. However, the question remains if physical exercise is a general sign of good health with direct or indirect effects on maintenance or improvement of smell capacity.

As nasal polyp recurrence is a clinical reality in a significant number of CRSwNP patients, Qi et al. ${ }^{(8)}$ have been able to identify mRNA levels of Charcot Leyden Crystals (CLC) in nasal brushing samples as a non-invasive and predictive marker of nasal polyp recurrence. Higher levels of CLC were associated with a higher likelihood of polyp recurrence, occurring in nearly $50 \%$ of their patients within 12-18 months after surgery. In a 5 year prospective trial comparing the outcomes of classic endoscopic sinus surgery (ESS) with more radical ESS with/ without Draf 3 procedure ${ }^{\left({ }^{9}\right)}$, radical approaches showed better outcomes. Of note, combining Draf 3 procedure with radical ESS did not yield better outcomes. Despite the interest of these data, they should be interpreted with caution given the small scale of the study and multiple variants that might influence the outcomes.

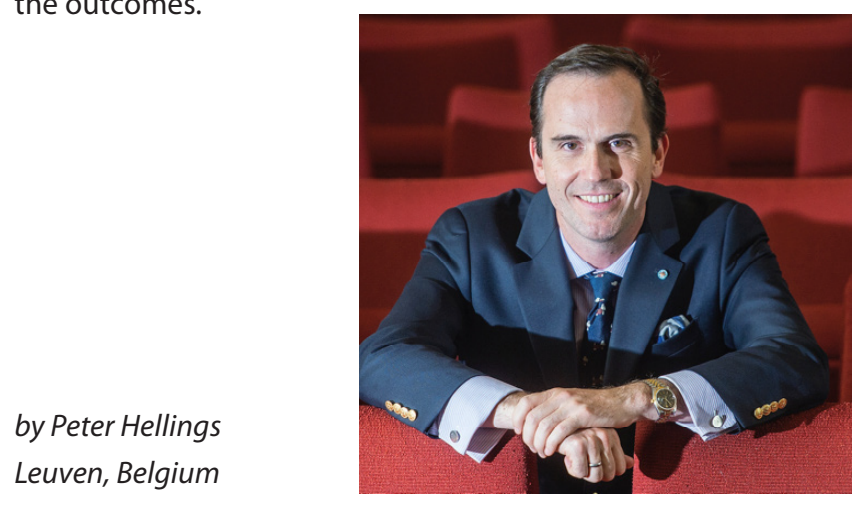

\section{References}

1. Fokkens WJ, et al. European Position Paper on Rhinosinusitis and Nasal Polyps 2020. Rhinology. 2020 Suppl. 29: 1-464.

2. Wahid N, et al. The socioeconomic cost of chronic rhinosinusitis study Rhinology. 2020;58(2):112-125

3. Forster-Ruhrmann $U$, et al., Rhinology. Rhinology. 2020 Feb 20. doi: 10.4193/ Rhin 19.318. [Epub ahead of print].

4. Ohki Y, et al. Local fungus-specific Immuno- globulin E production in chronic rhinosinusitis with nasal polyps. Rhinology. 2020;58(2): 136-144

5. Stöbauer J, et al. Prevalence and risk factors of smell dysfunction - a comparison between five German population-based studies. Rhinology. 2020;58(2): 184-193.

6. Lötsch J, et al. A data science-based analysis of seasonal patterns in outpatient presentations due to olfactory dysfunction Rhinology. 2020;58(2): 151-157.
7. Zhang C, et al. Role of physical exercise type in olfactory deterioration in ageing. Rhinology. 2020;58(2):145-150

8. Qi S, et al. Predictive significance of Charcot-Leyden Crystal mRNA levels in nasal brushing for nasal polyp recurrence. Rhinology. 2020;58(2):166-174.

9. Zhang $L$, et al. Long-term outcomes of different endoscopic sinus surgery in recurrent chronic rhinosinusitis with nasal polyps and asthma Rhinology. 2020;58(2):126-135. 